\title{
A Design of Microstrip-Disk-Type Directional Couplers
}

\author{
Tadashi Kawai, Takeshi Fukumoto, Yoshihiro Kokubo, Isao Ohta, and Hsu, Jui-Pang ${ }^{\dagger}$ \\ Department of Electronics, Faculty of Engineering, Himeji Institute of Technology \\ 2167 Shosha, Himeji-shi, Hyogo, 671-22 Japan \\ $\dagger$ Department of Electrical Engineering, Faculty of Engineering, Kanagawa University \\ Kanagawa-ku, Yokohama, 221 Japan
}

\begin{abstract}
This paper describes a design of microstrip rectangular disk quadrature directional couplers on the basis of a modified eigenfunction expansion method with consideration for frequency dependences of an effective width and an effective relative permittivity of a microstrip disk circuit. Using this approximate method, we can optimize a disk configuration of directional couplers in a reasonable computational time. The validity of the design method is examined by comparison with the numerical results by the FDTD method.
\end{abstract}

\section{INTRODUCTION}

Directional couplers and hybrids are very important passive components in microwave and millimeter-wave circuit systems. Planar-circuit-type quadrature hybrids in a triplate structure have beea proposed, and good characteristics have been obtained [1].

This paper presents a convenient design method of microstrip-disk-type directional couplers based on a modified eigenfunction expansion method with consideration for frequency dependences of the effective strip width and the relative permittivity.

Next, the validity of this approximate analysis is examined by the use of the Finite Difference Time Domain method (FDTD method). As a result, we can find the present design method to be useful for optimizing a disk configuration in a short time. Therefore, the approximate design method presented here is efficient to design directional couplers consisting of a microstrip disk.

\section{CIRCUIT CONFIGURATION AND DESIGN METHOD}

Fig. 1 shows the two circuit configurations to be considered. These circuits possess twofold symmetry with respect to the planes $\mathrm{AA}^{\prime}$ and $\mathrm{BB}^{\prime}$, and hence can be analyzed by reducing the four-port networks to four kinds of one-port circuits, that is, four quarter-circuits cut out of each four-port network along the

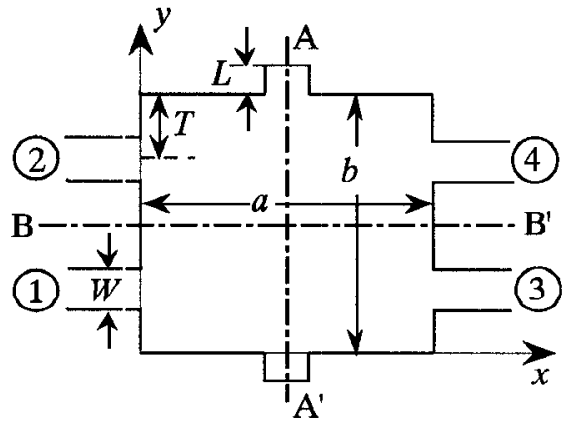

(a)

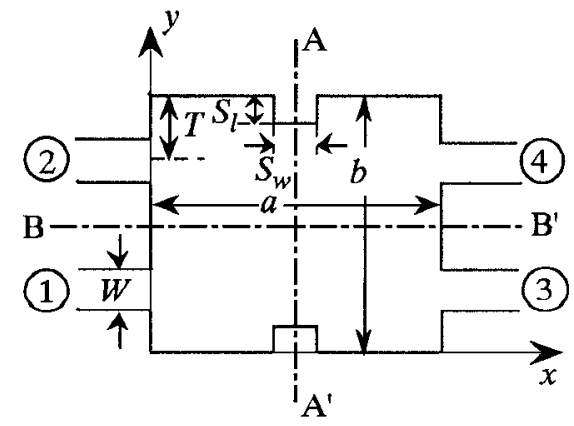

(b)

Fig. 1 Circuit configurations. (a) Convex version. (b) Concave version. 


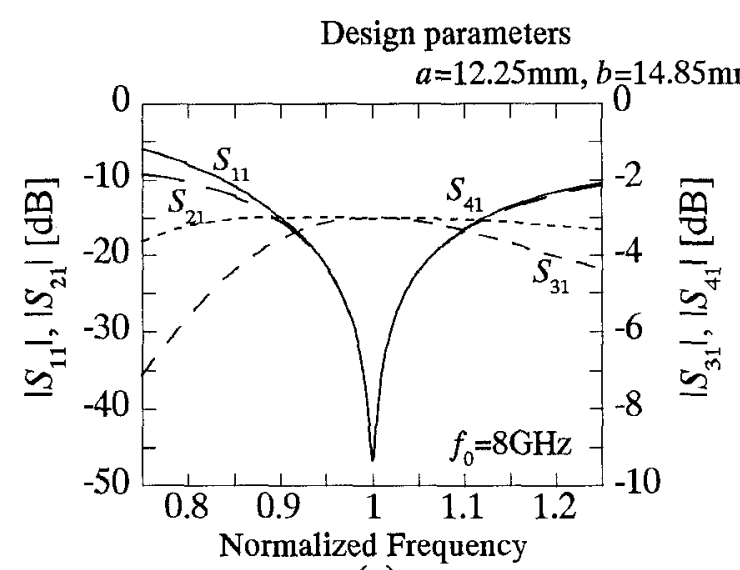

(a)

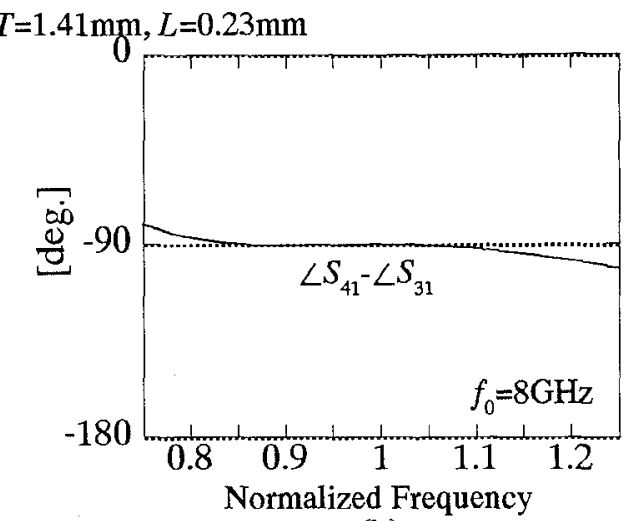

(b)

Fig. 2 Optimum design parameters and frequency characteristics of scattering parameters of the coupler with disk configuration in Fig. 1(a) designed by a modified eigenfunction expansion method. (a) Magnitudes and (b) phase difference between $S_{41}$ and $S_{31}$.

planes $A A^{\prime}$ and $\mathrm{BB}^{\prime}$ with a magnetic or electric boundary. When the reflection coefficients of the four one-port circuits, in other words, the eigenvalues of the scattering matrix are denoted by $s_{i}$ $(i=1,2,3,4)$, the scattering matrix of the original four-port circuit is given as [2]

$$
S=\left[\begin{array}{llll}
\alpha & \beta & \gamma & \delta \\
\beta & \alpha & \delta & \gamma \\
\gamma & \delta & \alpha & \beta \\
\delta & \gamma & \beta & \alpha
\end{array}\right]
$$

where

$$
\begin{aligned}
& \alpha=\left(s_{1}+s_{2}+s_{3}+s_{4}\right) / 4 \\
& \beta=\left(s_{1}+s_{2}-s_{3}-s_{4}\right) / 4 \\
& \gamma=\left(s_{1}-s_{2}+s_{3}-s_{4}\right) / 4 \\
& \delta=\left(s_{1}-s_{2}-s_{3}+s_{4}\right) / 4 .
\end{aligned}
$$

In addition, the subscript $i$ corresponds to an even or odd excitation with respect to the two planes, i.e., planes $\mathrm{AA}^{\prime}$ and $\mathrm{BB}^{\prime}$ are magnetic for $i=1$, $\mathrm{AA}^{\prime}$ electric and $\mathrm{BB}^{\prime}$ magnetic for $i=2, \mathrm{AA}^{\prime}$ magnetic and $\mathrm{BB}^{\prime}$ electric for $i=3$, and $\mathrm{AA}^{\prime}$ and $\mathrm{BB}^{\prime}$ electric for $i=4$.

The analysis is performed on the basis of the planar circuit concept. Although a Green's function cannot be written directly for the planar circuit shapes in Fig. 1, those circuits can be decomposed into regular shapes. Therefore the input impedance for one-port quarter circuits are derive by the segmentation method [3]. These eigenimpedances are transformed to $S$-matrix eigenvalues in the ordinary relation between the input impedance $Z_{i}$ and the reflection coefficient $s_{i}$ as follows:

$$
s_{i}=\frac{Z_{i}-Z_{0}}{Z_{i}+Z_{0}}
$$

where $Z_{0}$ represents the characteristic impedance of the input/output ports.

For a triplate-type circuit, a static approximation is sufficient to get its accuracy characteristics [4]. On the other hand, for the microstrip-type one, it is insufficient because of the imbalance of a circuit construction. Therefore, we consider a frequency-dependence of the effective width of a strip conductor as following equation [5], [6]:

$$
\begin{aligned}
& W_{\text {off }}(f)=W+\left(W_{\text {off } 0}-W\right) /\left(1+f / f_{g}\right) \\
& f_{g}=c_{0} /\left(2 W \sqrt{\varepsilon_{r}}\right)
\end{aligned}
$$

where $W$ and $W_{\text {eff }}$ are a physical and a static ef- 
fective width of the strip conductor, $f, c_{0}, \varepsilon_{r}$ represent frequency in $\mathrm{GHz}$, light velocity, and relative permittivity of the dielectric substrate, respectively. Furthermore, that of the effective relative permittivity is given by [7]

$$
\varepsilon_{r e f f}(f)=\varepsilon_{r}-\frac{\varepsilon_{r}-\varepsilon_{e f f}}{1+\left(f / f_{a}\right)^{m}}
$$

where

$$
\begin{aligned}
& \varepsilon_{r f f}=\frac{\varepsilon_{r}+1}{2}+\frac{\varepsilon_{r}-1}{2}\left(1+\frac{12 d}{W}\right)^{-1 / 2}+F\left(\varepsilon_{r}, d\right) \\
& F\left(\varepsilon_{r}, d\right)= \begin{cases}0.02\left(\varepsilon_{r}-1\right)\left(1-\frac{W}{d}\right)^{2}, & \frac{W}{d}<1 \\
0 \quad & \frac{W}{d}>1\end{cases} \\
& f_{a}=\frac{f_{b}}{0.75+\left(0.75-0.332 \varepsilon_{r}^{-1.73}\right) W / d} \\
& f_{b}=\frac{47.746}{d \sqrt{\varepsilon_{r}-\varepsilon_{r e f f}}} \tan ^{-1} \varepsilon_{r} \sqrt{\frac{\varepsilon_{r e f 0}-1}{\varepsilon_{r}-\varepsilon_{r e f 0}}} \\
& m= \begin{cases}m_{0} m_{c}, & m_{0} m_{c}<2.32 \\
2.32, & m_{0} m_{c} \geq 2.32\end{cases} \\
& m_{0}=1+\frac{1}{1+\sqrt{W / d}}+0.32(1+\sqrt{W / d})^{-3} \\
& m_{\mathrm{c}}= \begin{cases}1+\frac{1.4}{1+W / d}-\left(0.15-0.235 e^{-0.05 / f / 4}\right), & W / d \leq 0.7 \\
1+ & W / d>0.7\end{cases}
\end{aligned}
$$

where $\varepsilon_{\text {effo }}$ indicates a static effective permittivity, and $d$ and $\varepsilon_{r}$ a thickness and a relative permittivity of the dielectric substrate, respectively.

Then we try to analyze and design a directional coupler by expanding a Green's function in terms of eigenfunctions with eqs. (3) to (7). In this design, $\varepsilon_{r}$ and $d$ are chosen as 2.53 and $0.765 \mathrm{~mm}$, respectively. As a mathematical technique for optimization, Powell's method was employed [7]. Fig. 2 shows the theoretical scattering parameters of a coupler with an equal

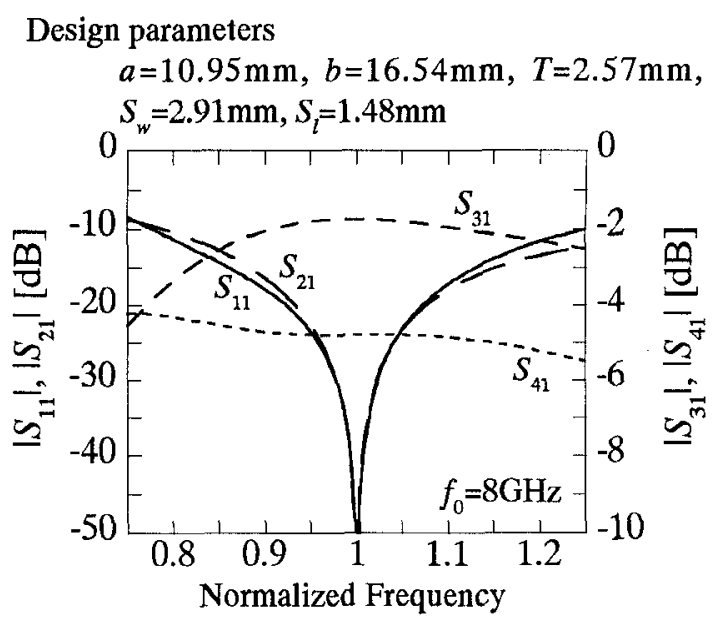

Fig. 3 Theoretical magnitudes of $S$-parameters as a function of frequency for the circuit shown in Fig. 1(b) with a power-split ratio of 2 .

power-split ratio $\left(R=\left|S_{31} / S_{41}\right|^{2}=1\right)$ designed at a center frequency of $8 \mathrm{GHz}$ using a convex-version in Fig. 1(a). The relative bandwidth is about 12.5 percent with a tolerance limit for return loss and isolation of $20 \mathrm{~dB}$. Next we try to design couplers with unequal power-split ratios using the concave-version in Fig. 1(b). Fig. 3 exhibits the magnitude of $S$-parameters of a coupler with $R=2$. We can design couplers within the powersplit ratio of about 1 to 5 using the circuit configurations shown in Fig. 1.

\section{ANALYSIS BY FDTD METHOD}

In order to confirm the approximate analysis in the previous section, we apply the method of FDTD [9] to the couplers and analyze the circuit property strictly. Fang's third order absorbing boundary conditions are used [10]. Fig. 4 shows the calculated $S$-parameters of the $3 \mathrm{~dB}$ coupler shown in Fig. 2. This result is nearly agreement with the above approximate analysis.

\section{CONCLUSIONS}

A simple design method of the microstrip 


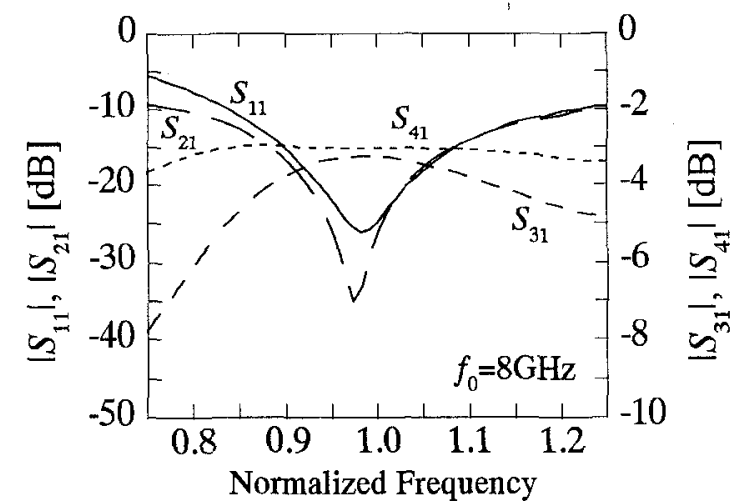

(a)

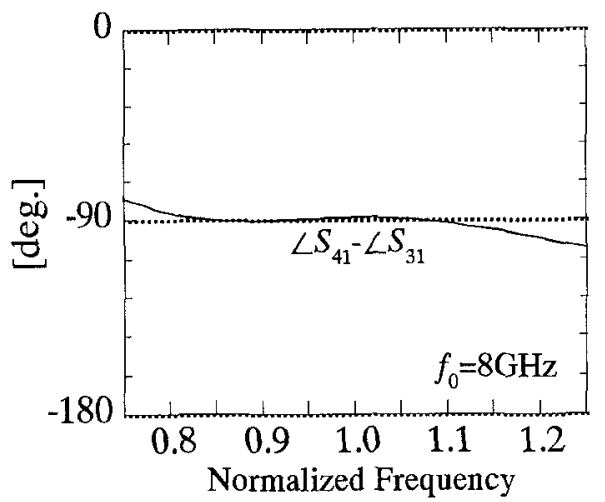

(b)

Fig. 4 Frequency characteristics of $S$-parameters of the coupler in Fig.2 computed by the FDTD method. (a) Magnitudes and (b) phase difference between $S_{41}$ and $S_{31}$.

rectangular disk directional couplers has been described on the basis of a modified eigenfunction expansion method with consideration for frequency dependences of the effective strip width and the effective relative permittivity. Furthermore, this approximate analysis has been confirmed through numerical results by use of the FDTD method. This method enables us to simply design microstrip-disk-type couplers in a short computational time.

\section{REFERENCES}

[1] T. Kawai and I. Ohta :"Planar-circuit-type 3-dB quadrature hybrids," IEEE Trans. Microwave Theory Tech., MTT-42, 12, pp.2462-2467, Dec. 1994.

[2] C. G. Montgomery, R. H. Dicke, and E. M. Purcell, Principle of Microwave Circuits. New York: McGraw-Hill, 1948.

[3] R. Chadha and K. C. Gupta :"Segmentation method using impedance matrices for analysis of planar microwave circuits," IEEE Trans. Microwave Theory Tech., MTT-29, 1, pp.71-74, Jan. 1981.

[4] T. Okoshi : "Planar Circuits," pp.32-33, Springer-Verlag, Berlin, 1985.

[5] E. Yamashita, K. Atsuki, and T. Umeda: "An approximate dispersion formula of microstrip lines for computer-aided design of microwave integrated circuits," IEEE Trans. Microwave Theory Tech., MTT-27, 12, pp.1036-1038, Dec. 1979.

[6] G. Kompa and R. Mehran: "Planar waveguide model for calculating microstrip components," Electronics Letter, 11, pp.459460,1975 .

[7] R. E. Collin, Foundations for Microwave Engineering (Second Edition). pp.136-163, New York: McGraw-Hill, 1992.

[8] M. J. D. Powell : "An efficient method for finding the minimum of a function of several variables without calculating derivatives," Computer J., 7, pp.155-162, July 1964.

[9] K. S. Yee : "Numerical solution of initial boundary value problems involving Maxwell's equations in isotropic media," IEEE Trans. Antennas Propagation, AP-14, pp.302-307, May 1966.

[10]J. Fang :"Absorbing boundary conditions applied to model wave propagation in microwave integrated-circuits," IEEE Trans. Microwave Theory Tech, MTT-42, 8, pp.1506-1513, August 1994. 